\title{
Dementia is Associated with a Syndrome of Global Neuropsychiatric Disturbance
}

\begin{abstract}
Donald R. Royall, M.D. Departments of Psychiatry, Medicine, and Family and Community Medicine, and the Biggs Institute for Alzheimer's and Neurodegenerative Disorders at the University of Texas Health Science Center, San Antonio, TX TEL: (210) 567-1255, FAX:(210) 5671269 royall@uthscsa.edu
\end{abstract}

\author{
Raymond F. Palmer, Ph.D. \\ Department of \\ Family and Community Medicine, \\ the University of Texas Health Science \\ Center, San Antonio, TX \\ TEL: (210) 358-3883, \\ palmerr@uthscsa.edu
}

\section{and the Alzheimer's Disease Neuroimaging Initiative*}

*Some data used in preparation of this article were obtained from the Alzheimer's Disease Neuroimaging Initiative (ADNI) database (adni.loni.usc.edu). ADNI had no role in study design, data collection and analysis, decision to publish, or preparation of the manuscript. However, ADNI investigators contributed to the design and implementation of $\mathrm{ADNI}$ and/or provided data. A complete listing of $\mathrm{ADNI}$ investigators can be found at: http://adni.loni.usc.edu/wpcontent/uploads/how_to_apply/ADNI_Acknowledgement_List.pdf.

Key words: BPSD, Cognition, Dementia, Functional Status, $g$, intelligence

${ }^{*}$ Corresponding Author:

${ }^{*}$ Donald R. Royall, M.D.

Department of Psychiatry

University of Texas Health Science Center at San Antonio 7703 Floyd Curl Dr.

San Antonio, TX 78229-3900

TEL: (210) 567-1255

FAX:(210) 567-1269

Email: royall@uthscsa.edu 


\section{Acknowledgments:}

This work was supported by the Julia and Vann Buren Parr endowment for the study of Alzheimer's Disease. The funders had no role in study design, data collection and analysis, decision to publish, or preparation of the manuscript.

Some data used in preparation of this article were obtained from the ADNI database (adni.loni.usc.edu). Data collection and sharing for this project was funded by the Alzheimer's Disease Neuroimaging Initiative (ADNI) (National Institutes of Health Grant U01 AG024904) and DOD ADNI (Department of Defense award number W81XWH-12-2-0012). ADNI is funded by the National Institute on Aging, the National Institute of Biomedical Imaging and Bioengineering, and through generous contributions from the following: AbbVie, Alzheimer's Association; Alzheimer's Drug Discovery Foundation; Araclon Biotech; BioClinica, Inc.; Biogen; Bristol-Myers Squibb Company; CereSpir, Inc.; Cogstate; Eisai Inc.; Elan Pharmaceuticals, Inc.; Eli Lilly and Company; Eurolmmun; F. Hoffmann-La Roche Ltd and its affiliated company Genentech, Inc.; Fujirebio; GE Healthcare; IXICO Ltd.; Janssen Alzheimer Immunotherapy Research \& Development, LLC.; Johnson \& Johnson Pharmaceutical Research \& Development LLC.; Lumosity; Lundbeck; Merck \& Co., Inc.; Meso Scale Diagnostics, LLC.; NeuroRx Research; Neurotrack Technologies; Novartis Pharmaceuticals Corporation; Pfizer Inc.; Piramal Imaging; Servier; Takeda Pharmaceutical Company; and Transition Therapeutics. The Canadian Institutes of Health Research is providing funds to support ADNI clinical sites in Canada. Private sector contributions are facilitated by the Foundation for the National Institutes of Health (www.fnih.org). The grantee organization is the Northern California Institute for Research and Education, and the study is coordinated by the Alzheimer's Therapeutic Research Institute at the University of Southern California. ADNI data are disseminated by the Laboratory for Neuro Imaging at the University of Southern California.

This analysis has been presented as an abstract at the Gerontological Society of America's 71st Annual Scientific Meeting in Austin, Texas on November 16, 2019. 


\section{Abstract: (268 words)}

67 Objective: Global factors have been identified in measures of cognitive performance

68 (i.e., Spearman's g) and psychopathology (i.e., "General Psychopathology", "p").

69 Dementia is also strongly determined by the latent phenotype " $\delta$ ", derived from $g$. We

70 wondered if the Behavior and Psychological Symptoms of Dementia (BPSD) might arise

71 from an association between $\delta$ and $p$.

72 Methods: $\delta$ and $p$ were constructed by confirmatory factor analyses in data from the

73 Alzheimer's Disease Neuroimaging Initiative (ADNI). $\delta$ and orthogonal factors

74 representing "domain-specific" variance in memory (MEM) and executive function (EF)

75 were regressed onto $p$ and orthogonal factors representing "domain-specific" variance in

76 positive (+) and negative (-) symptoms rated by the Neuropsychiatric Inventory Nursing

77 Home Questionnaire (NPI-Q) by multiple regression in a structural equation model

78 (SAM) framework.

79 Results: Model fit was excellent $(C F I=0.98, R M S E A=0.03) . \delta$ was strongly associated

80 with $p,(+)$ and $(-)$ and strongly associated with $p(r=-0.57, p<0.001)$. All three

81 associations were inverse (adverse). Independently of $\delta$, MEM was uniquely associated

82 with (+), while ECF was associated with (-). Both associations were moderately strong.

83 ECF was also weakly associated with $p$.

84 Conclusions: Dementia severity $(\delta)$ derived from general intelligence $(g)$ is specifically

85 associated with general psychopathology $(p)$. This is $p$ 's first demonstration in an elderly

86 sample and the first to distinguish the global behavioral and psychological symptoms

87 specific to dementia (BPSSD) from behavioral disturbances arising by way of non-

88 dementing, albeit likely disease-specific, processes affecting domain-specific cognitive

89 and behavioral constructs. Our findings call into question the utility of proposed regional 
bioRxiv preprint doi: https://doi.org/10.1101/813485; this version posted October 21,2019 . The copyright holder for this preprint (which was not certified by peer review) is the author/funder, who has granted bioRxiv a license to display the preprint in perpetuity. It is made available under aCC-BY-NC-ND 4.0 International license.

90 interventions in BPSSD, and point to the need to explore global interventions against

91 dementia-specific behavioral features.

92

93

94 


\section{Introduction:}

97 The so-called Behavioral and Psychological Symptoms of Dementia (BPSD) are a major

98 source of comorbidity and caregiver burden (1). BPSD increase exposure to

99 psychopharmacological agents and their associated adverse drug reactions (ADR) (2).

100 They are a major factor in caregiver stress (3-4), increase the risk of institutionalization

101 (5) and inflate the costs associated with dementia care (3).

103 Regardless, surprisingly little is known about the biological substrates of BPSD, their 104 natural history or risk factors. It is widely assumed that BPSD arise from the regional 105 neuropathology(ies) specific to the disease(s) in which they develop. Some investigators 106 have attempted to use to BPSD to develop etiologically precise BPSD signatures. 107 However, in young adults, a global "general psychpathology" factor " $p$ " has been 108 proposed to influence all psychopathological behavioral domains (6-7) and is 109 demonstrable in a wide range of conditions (8).

$111 p$ has never been addressed in the context of dementia and BPSD. However, it 112 recapitulates Spearman's well-accepted general intelligence factor " $g$ " (9). Our recent

113 work strongly implicates $g$ as the essential cognitive determinant of dementia severity 114 (10). Only via $g$ can cognitive performance be meaningfully related to functional status.

115 We have demonstrated this via theory-driven bi-factor confirmatory analyses (CFA) in a 116 Structural Equation Model (SEM) framework (11-12). The resulting latent variable "ס" (for 117 "dementia") is strongly associated with the Clinical Dementia Rating Scale (CDR) "Sum 118 of boxes" (CDR-SB) (13), cross-sectionally (14), longitudinally (15-16), and across 119 diagnoses (16). 
$121 \delta$ is highly accurate in distinguishing demented cases (16), but it is entirely "agnostic" as

122 to dementia's etiology (17). If $p$ can be shown to be associated specifically with $\delta$ then it

123 would directly implicate dementia itself as the transdiagnostic determinant of global

124 psychopathology, link $p$ to intelligence, and constrain $p$ 's biology to that of $g$ and $\delta$.

126 Conversely, "domain-specific" variance in cognitive performance (i.e., "memory", "frontal

127 lobe functions", etc.) is "orthogonal" (unrelated) to $g(11-12)$. It therefore risks to be

128 unrelated to both $\delta$ and IADL, and thus to be independent of dementia per se. In this

129 study, we test the fit of $p$ to BPSD reported participants in the Texas Alzheimer's

130 Research and Care Consortium (TARCC) and relate cognitive performance, including $\delta$

131 and orthogonal domain-specific factors, to prospective variance in $p$ and orthogonal

132 domain-specific behavioral clusters.

\section{Methods:}

136 Subjects:

138 Subjects included $n=3385$ participants in the Texas Alzheimer's Research and Care

139 Consortium (TARCC). The Consortium's methods have been described in detail

140 elsewhere (18). Briefly, the TARCC cohort is a convenience sample of well-

141 characterized cases of Alzheimer's disease (AD) ( $n=1240)$, "Mild Cognitive Impairment

142 "(MCl) ( $\mathrm{n}=688)$, and normal controls $(\mathrm{NC})(\mathrm{n}=1384)$. Each TARCC participant

143 undergoes a standardized annual examination that includes a medical evaluation,

144 neuropsychological testing, and clinical interview. Diagnosis of AD is based on National

145 Institute for Neurological Communicative Disorders and Stroke-Alzheimer's Disease and

146 Related Disorders Association (NINCDS-ADRDA) criteria. All TARCC evaluations and 
147 psychometrics are provided in English or in Spanish according to the subject's

148 preference. Institutional Review Board approval was obtained at each site and written

149 informed consent was obtained for all participants.

\section{Statistical Analyses:}

153 This analysis was performed using Analysis of Moment Structures (AMOS) software 154 (19). The maximum likelihood estimator was chosen. Co-variances between the 155 residuals were estimated if they were significant and improved fit.

157 Neuropsychiatric Indicators:

159 The Neuropsychatric Inventory (NPIQ) (20): Behavioral and neuropsychiatric

160 disturbances were measured at Wave 2 by the informant-rated NPI-Q. Multiple Spanish

161 translations are available. We used the Spanish version generated by the Spanish

162 Translation and Adaptation Work Group (STAWG) from the National Alzheimer's

163 Coordinating Center (NACC) Uniform Data Set (UDS) (21). This scale has been well-

164 validated among Mexican American (MA) respondents (22).

166 The NPI-Q encompasses 12 behavioral features that are commonly exhibited in the 167 context of neuropsychiatric illness. For this study, a modified NPIQ was administered by 168 an experienced research assistant to knowledgeable informants (caregivers), who were 169 asked to rate the presence and the severity of each BPSD, i.e., agitation/aggression, 170 dysphoria/depression, irritability/lability, apathy/indifference, anxiety, disinhibition, 171 aberrant motor behavior, delusions, hallucinations, euphoria/ elation, nighttime 172 behavioral disturbances, and appetite/eating disturbances. 
174 Informants were first asked to endorse the presence or absence of each BPSD over the

175 prior four weeks, using a single screening question. If the informant endorsed the

176 behavior, a severity score was assigned on a three-point Likert scale (i.e., 1-mild, 2-

177 moderate, 3-severe). If the informant did not endorse a behavior, its "severity" was rated

178 "zero". Thus, in our modified adaptation, each NPI-rated BPSD ranged from zero (not

179 endorsed) to 3 "severe". The total NPI-Q severity score represents the sum of the 12

180 individual modified symptom severity scores and ranges from 0 to 36 (NPI-QTOT).

182 Covariates:

183 All observed measures in the structural models were adjusted for baseline age, body

184 mass index (BMI), education, ethnicity, gender, Mini-Mental Status Exam (MMSE) score

\section{5 (23), HCY, and HgbA1c.}

187 Age: Self-reported age was confirmed by birthdate and coded continuously.

189 Body Mass Index (BMI): BMI was estimated as the ratio of subject height to weight 190 (REF).

192 Education: Education was coded continuously as years of formal education.

194 Ethnicity: Ethnicity was determined by self-report and coded dichotomously as

195 "Hispanic" = 1 and "non-Hispanic White" $(\mathrm{NHW})=0$.

196 Gender: Gender was coded dichotomously with "female" = 1. 
198 The Clinical Dementia Rating Scale sum of boxes (CDR-SB) (24): The Clinical Dementia

199 Rating Scale "sum of boxes (CDR-SB) (13) The CDR is used to evaluate dementia

200 severity. The rating assesses the patient's cognitive ability to function in six domains -

201 memory, orientation, judgment and problem solving, community affairs, home and

202 hobbies and personal care. Information is collected during an interview with the patient's

203 caregiver. Optimal CDR-SB ranges corresponding to global CDR scores are $0.5-4.0$ for

204 a global score of $0.5,4.5-9.0$ for a global score of 1.0, $9.5-15.5$ for a global score of

2052.0 , and $16.0-18.0$ for a global score of 3.0 .

207 The Geriatric Depression Rating Scale (GDS) (25): GDS scores range from zero-30.

208 Higher scores are worse. A cut-point of 9-10 best discriminates clinically depressed from

209 non-depressed elderly.

211 The Mini-Mental Status Examination (MMSE) (23): The MMSE is a well-known and

212 widely used test for cognitive impairment screening. Scores range from 0 to 30 . Scores

213 less than 24 reflect cognitive impairment.

Analysis Sequence:

217 The NPI-Q items' severity ratings were submitted to a CFA in SEM. First, a latent

218 variable " $p$ " was derived from all 12 BPSD. Orthogonal latent factors rating "positive" (+)

219 and negative (-) domain-specific symptom clusters were constructed from p's residuals.

220 This renders (+) and (-) "orthogonal" (unrelated) to $p$ because their respective indicators

221 are already adjusted for that global construct. The result is a "bifactor" CFA model

222 defining $p$ and two orthogonal domain-specific factors, (+) and (-) (Figure 1). 
224 The bifactor model's fit was compared to several alternatives, beginning with the single

225 factor $p$. In a second alternative, (+) and (-) were used as $p$ 's indicators, resulting in a

226 hierarchical model. Finally, all three models containing $p$ were compared to a previously

227 described four factor model which lacked this construct. It was developed by an

228 exploratory factor analysis of NPI-Q data (26). All four alternative models were

229 constructed from NPI-Q data collected at wave 2.

231 The best fitting moldel was associated with cognitive performance data collected at wave

2321 in TARCC (baseline). Now, cognitive performance data were submitted to a four factor

233 CFA as previously described (12). This results in the "dDx" $\delta$ homolog, and three

234 orthogonal factors, i.e., g' (dDx's residual in Spearmans's g) and two domain-specific

235 cognitive factors rating memory (MEM) and executive function (EF). In TARCC, the dDx

236 homolog has been reported to have a high area under the receiver operating

237 characteristic curve (AUC /ROC) for Alzheimer Disease's (AD)'s discrimination from

238 normal controls $(\mathrm{NC})(\mathrm{c}=0.98)$ and to be strongly associated with dementia severity as

239 measured by the CDR-SB $(r=0.88)$.

$241 \mathrm{dDx}, \mathrm{MEM}$ and EF at wave 1 were regressed onto wave $2 p,(+)$ and (-) by multivariate

242 regression in a structural SEM framework. The result is a multivariate regression model

243 of the baseline cognitive factors as predictors of prospective global and domain-specific

244 BPSD (Figure 2).

246 Missing data:

247 We used the newest instance of TARCC's dataset (circa 2016). The entire dataset was

248 employed. Clinical diagnoses were available on 3385 subjects, 2861 of whom had

249 complete data for $\delta$ 's cognitive indicators and covariates. Modern Missing Data Methods 
250 were automatically applied by the AMOS software (27). AMOS employs Full information

251 Maximum Likelihood (FIML) (28).

253 Fit indices:

254 Fit was assessed using four common test statistics: chi-square, the ratio of the chisquare

255 to the degrees of freedom in the model (CMIN /DF), the comparative fit index (CFI), and

256 the root mean square error of approximation (RMSEA). A non-significant chisquare

257 signifies that the data are consistent with the model (29). However, in large samples, this

258 metric conflicts with other fit indices (insensitive to sample size) show that the model fits

259 the data very well. A CMIN/DF ratio < 5.0 suggests an adequate fit to the data (30). The

260 CFI statistic compares the specified model with a null model (31). CFI values range from

2610 to 1.0 . Values below 0.95 suggest model misspecification. Values approaching 1.0

262 indicate adequate to excellent fit. An RMSEA of 0.05 or less indicates a close fit to the

263 data, with models below 0.05 considered "good" fit, and up to 0.08 as "acceptable“(32).

264 All fit statistics should be simultaneously considered when assessing the adequacy of

265 the models to the data.

267 Results:

269 The demographic characteristics of TARCC's sample are presented in Table 1.

271 Base model fit was excellent $(\mathrm{CFI}=0.98, \mathrm{RMSEA}=0.02)$. All behaviors loaded

272 significantly on $p$. The ad hoc (+) and (-) factors were significantly indicated by their

273 respective BPSD. The base model had improved fit over all alternatives. 
275 The base model's three BPSD factors, $p,(+)$ and (-), were regressed onto baseline

276 psychometric performance. Model fit was again excellent $(C F I=0.98, R M S E A=0.03) . \delta$

277 was strongly associated with $\mathrm{p},(+)$ and (-). All three associations were inverse (adverse).

278 Independently of $\delta$, MEM was uniquely associated with (+), while ECF was associated

279 with (-). Both associations were moderately strong. ECF was also weakly associated

280 with $p$.

281

282 Discussion: This analysis demonstrates a global BPSD factor's relatively good fit to 283 observed BPSD and p's statistically strong association with dementia severity as

284 measured by $\delta$. Additionally, MEM was associated specifically with (+), while ECF was

285 associated with (-), and with dementia.

287 Since $\delta$ and $g$ are both "indifferent to their indicators", they manifest in a wide range of 288 cognitive performance measures. We have demonstrated $\delta$ 's psychometric stability 289 across batteries, and even down to the item-set of an individual measure (33). The

290 spectrum of CNS-variables impacted by $\delta$ and $g$ may extend beyond cognition, into

291 sensory or even motor function. Spearman himself constructed a $g$ homolog from

292 sensory discrimination tasks alone (9).

294 Those findings appear to constrain $\delta$ 's (and therefore dementia's) biomarkers to those of

295 "intelligence" (Caveat: Since $g$ is manifest in all cognitive performance measures and 296 possibly in sensory and motor domains, "intelligence" in this context cannot be construed 297 as meaning "cleverness", "rationality" or "wisdom". Spearman's use of the word is limited 298 to a unique mathematical association, demonstrable by factor analysis). Dementia's true 
299 biomarkers must be similarly positioned to impact every cognitive performance measure.

300 Regional pathologies are disadvantaged in this regard.

302 The present findings suggest a similar constraint may relate to BPSD. Global 303 psychopathology (i.e., $p$ ) may relate to the globally distributed processes that impact $g$

304 through $\delta$. Domain-specific behavioral constructs, orthogonal to $p$, seem more likely

305 have regional determinants (34-35). This is consistent with our findings that MEM is

306 related to $(+)$ but not $(-)$, while ECF is related to (-) and not (+). MEM might be plausibly

307 associated with regional mesio-temporal pathology while ECF can be plausibly

308 associated with regional frontal circuit pathology. Replication of this analysis by the dT2A

309 homolog in ADNI (14) might allow for confirmation of such hypotheses against structural

310 and functional neuroimaging.

312 The possibility of a dementia-specific dysbehavioral syndrome was alluded to by

313 Hughlings Jackson in his 1884 description of the "dissolution" of the central nervous 314 system (CNS) (36). He argued for a hierarchical loss of neurological integrity that would 315 disrupt higher abilities and thereby "release" lower centers from control.

"I submit that disease only produces negative mental symptoms answering to the dissolution, and that all elaborate positive mental symptoms (illusions, hallucinations, delusions, and extravagant conduct)

322 Jackson further described "uniform" and "local" dissolutions. "In uniform dissolution the

323 whole nervous system is under the same conditions or evil influence, the evolution of the

324 whole nervous system is comparatively evenly reversed." He recognized that local 
325 pathologies arise from distinct etiologies and that "Different kinds of insanity are different

326 local dissolutions of the highest centres." This suggests that the behaviors arising from

327 global CNS disturbances might be a transdiagnostic property of dementing illness. The

328 regional disruptions unique to each dementing illness might engender diagnostically-

329 specific behavioral features, but those would not present transdiagnostically.

331 In this scenario, a global dysbehavioral syndrome would emerge in demented states

332 regardless of their etiology(ies) and in response to transdiagnostic pathology, not

333 disease-specific changes. Support for transdiagnostic dementia-specific behavioral

334 features is provided by the stereotyped natural history of BPSD at specific stages of

335 dementia's evolution (37), the transdiagnostic presentation of multiple BPSD (38-39) and

336 high penetrance of BPSD with increasing dementia severity (40). Support for a global vs.

337 "local" BPSD dichotomy can also be intuited in recent work on the bifactor nosology of

338 psychopathology in children and adults. Factor analyses of behavioral psychopathology

339 suggest independent effects of both global and domain-specific behavioral factors on

340 observed psychopathology (8).

342 Our model also suggests the potential for a novel rational approach to the treatment of

343 BPSD. BPSD arising from regional pathology(ies) and manifesting as domain-specific

344 behavioral issues may respond to "regional" pharmaco-biological interventions. BPSD

345 manifesting as $p$-related behavioral issues may require "global" interventions and /or

346 interventions directed at $\delta$ and its biomarkers.

347 Examples of regional interventions might include many traditional monoaminergic 348 approaches. Monoaminergic networks arise in the brainstem and project to regionally 349 precise targets in the neocortex and associated subcortical structures (41). They do not 350 project globally throughout the brain. Examples of monoaminergic interventions for 
351 BPSD might include atypical antipsychotics and Serotonin Selective Reuptake Inhibitor

352 (SSRI) antidepressants. Even-the pro cholinergic cholinesterase inhibitors are reported

353 to have efficacy against certain BPSD (42). More precisely localized regional

354 interventions would include regional lobotomy, deep-brain stimulation of specific

355 structures (e.g., the amygdala), and /or regional transcranial magnetic stimulation

356 (rTMS). All have been advocated for BPSB and related psychobehaviors in the literature

357 (43).

359 In contrast, y-aminobutyric acid (GABA) and glutamate are ubiquitously distributed, as

360 are the effects of hypoglycemia, seizure disorders, whole brain radiation, and possibly

361 blast-related traumatic brain injuries (TBI). Alcohol (EtOH) and benzodiazepines (BNZ)

362 may have GABA-mediated adverse effects on behavior and cognition manifesting as

363 changes in $g, \delta$ and $p$. The protean impacts of those insults beyond cognition and

364 behavior, including also balance, sensory and motor performance may betray effects on

$365 g, \delta$ and $p$. Thus, the BPSD-related to $g, \delta$ and $p$ may respond better to global

366 interventions, e.g., mood stabilizing anticonvulsants, lithium, and or electroconvulsive

367 therapies (ECT). Alternatively, $g, \delta$ and $p$ might be adversely impacted by interventions

368 of this class, explaining the insalubrious reputation of BNZ and $\mathrm{EtOH}$ in cognitively

369 impaired persons.

370

371 In summary, we have specifically associated general psychopathology $(p)$ with a latent

372 dementia severity metric $(\delta)$ derived from general intelligence $(g)$. This is $p$ 's first

373 demonstration in an elderly sample and the first to distinguish the global behavioral and

374 psychological symptoms specific to dementia (BPSSD) from behavioral disturbances

375 arising by way of non-dementing, albeit disease-specific processes affecting domain- 
bioRxiv preprint doi: https://doi.org/10.1101/813485; this version posted October 21,2019 . The copyright holder for this preprint (which was not certified by peer review) is the author/funder, who has granted bioRxiv a license to display the preprint in perpetuity. It is made available under aCC-BY-NC-ND 4.0 International license.

376 specific cognitive and behavioral constructs. Our findings call into question the utility of

377 proposed regional interventions in BPSSD, and point to the need to explore global

378 interventions against dementia-specific behavioral features.

379

380

381

382

383 


\section{Table 1: Descriptive Statistics}

385

\begin{tabular}{|l|c|c|}
\hline Variable & N & Mean (SD) \\
\hline Age (observed) & 3381 & $70.88(9.48)$ \\
\hline CDR (Sum of Boxes) & 3306 & $2.42(3.35)$ \\
\hline EDUC (observed) & 3381 & $13.24(4.25)$ \\
\hline Ethnicity $(1=\mathrm{MA}, \mathrm{n}=1189)$ & 3381 & $0.36(0.47)$ \\
\hline GDS ${ }_{30}$ (observed) & 3005 & $5.60(5.25)$ \\
\hline Gender $(\precsim=1, \mathrm{n}=1281)$ & 3312 & $0.39(0.49)$ \\
\hline IADL (Summed) & 3381 & $10.48(4.52)$ \\
\hline MMSE & 3311 & $25.52(4.76)$ \\
\hline Complete Cases & 2861 & \\
\hline
\end{tabular}

CDR = Clinical Dementia Rating scale; COWA = Controlled Oral Word Association Test; DIS = Digit Span Test GDS = Geriatric Depression Scale; IADL = Instrumental Activities of Daily Living; MMSE = Mini-mental State Exam; SD = standard deviation; WMS LM II = Weschler Memory Scale: Delayed Logical Memory; WMS VR I = Weschler Memory Scale: Immediate Visual Reproduction. 
395

396

397

398

\section{Table 2: Model Fit}

\begin{tabular}{|l|c|c|c|c|c|}
\hline Alternative Models & CHISQ & df & CFI & RSMEA & ACC \\
\hline 1 $p$ only & 551.55 & 54 & 0.92 & 0.05 & 623.55 \\
\hline 2 Hierarchical & 463.32 & 52 & 0.93 & 0.05 & 539.32 \\
\hline 3 Conventional (no $p)[R E F]$ & 2677.81 & 53 & 0.58 & 0.12 & 2751.81 \\
\hline $\begin{array}{l}\text { 4 Orthogonal bifactor } \\
{[p,(+),(-) \text { (Figure 1)] }}\end{array}$ & 196.35 & 42 & 0.98 & 0.03 & 292.35 \\
\hline
\end{tabular}




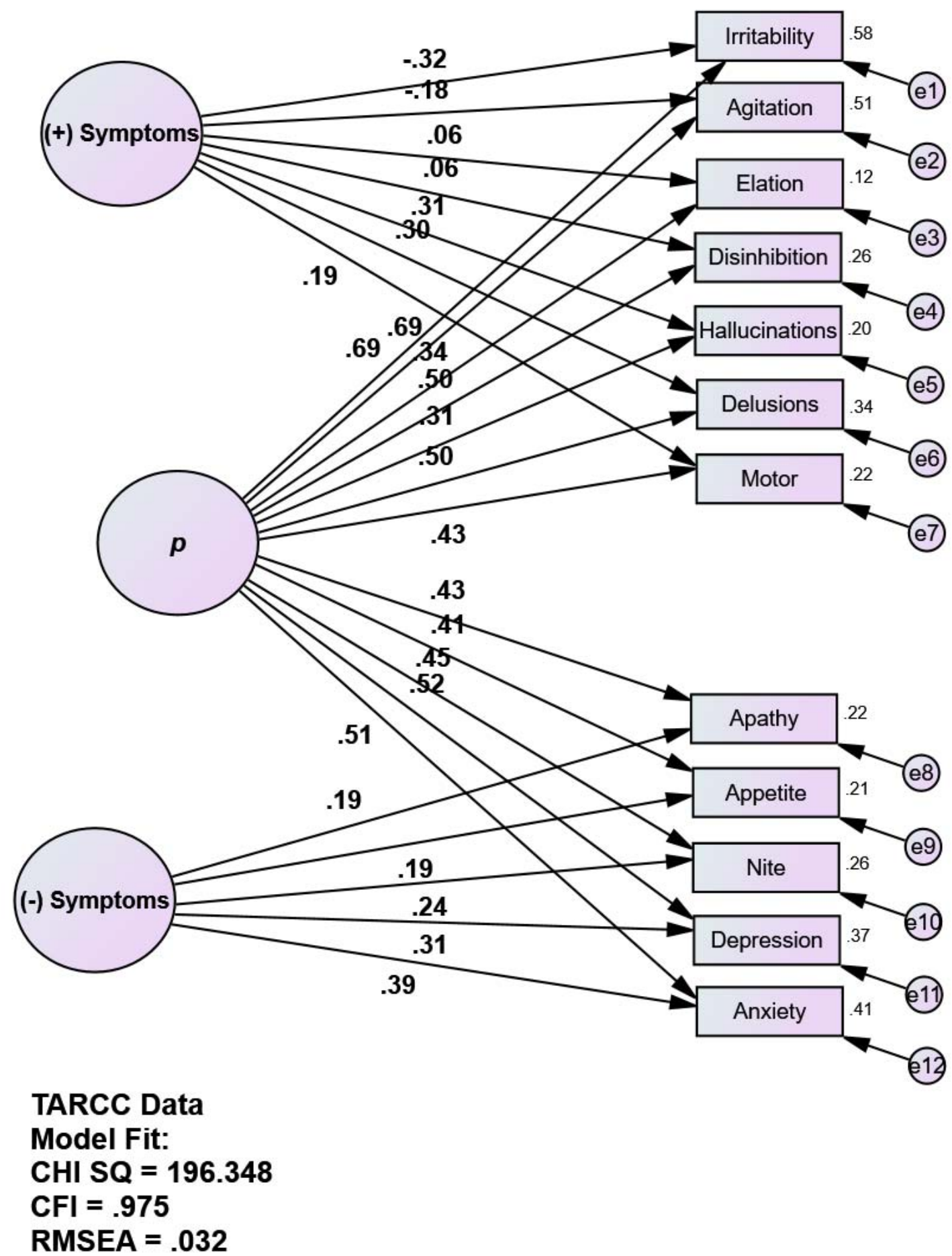

Index; BPSD = Behavior and Psychological Symptoms of Dementia; CFA = Confirmatory Factor Analysis; GDS = Geriatric Depression Scale; HCY = serum homocysteine; HgbA1c = serum hemoglobin A1c; RMSEA = Root Mean Square Error of Association.

*All observed variables except APOE are adjusted for age, education, ethnicity, gender, GDS, HCY, and HgbA1c (paths not shown for clarity). Those covariates are densely intercorrelated. 


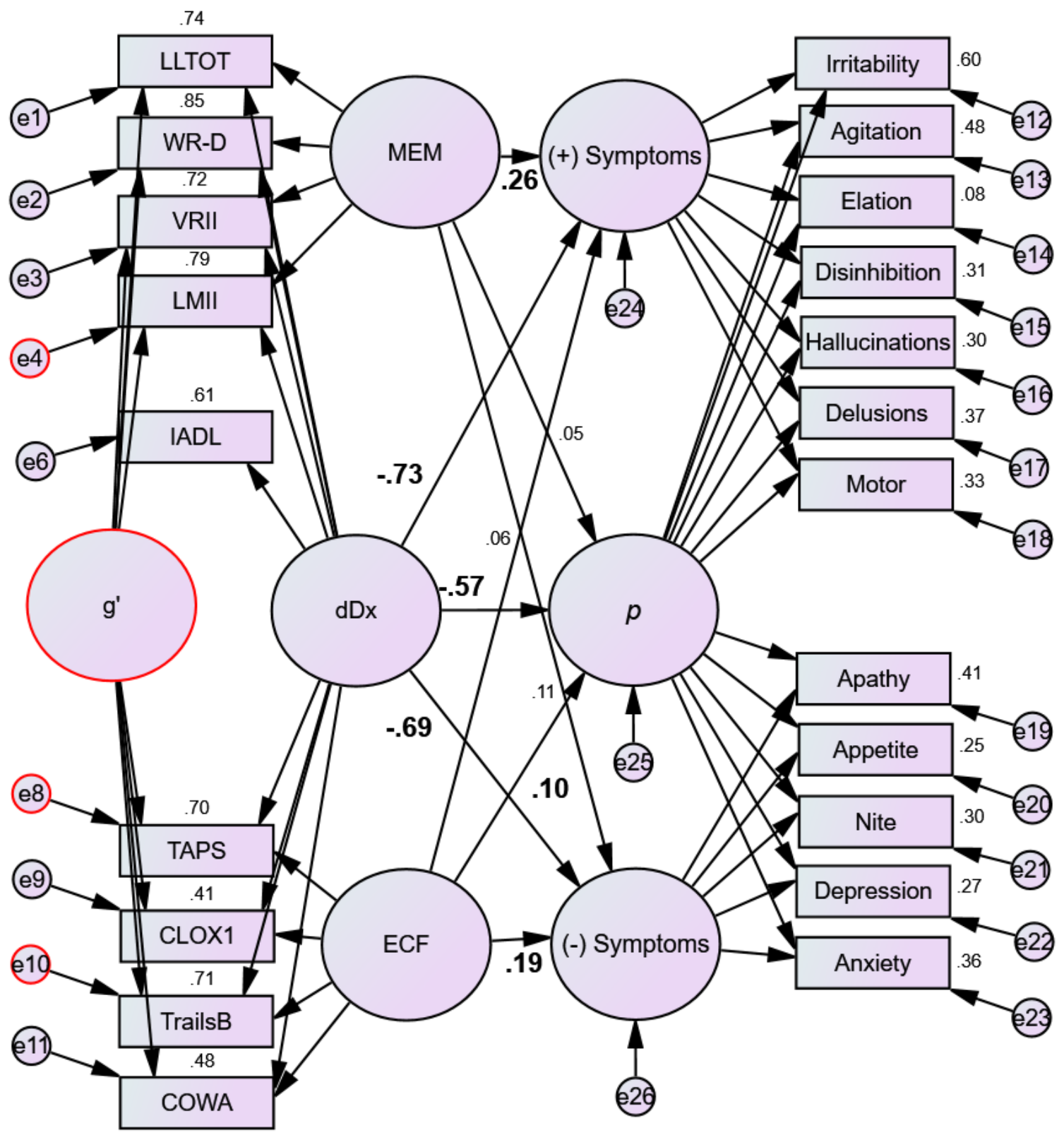

TARCC Data

Model Fit:

$\mathrm{CHI} S \mathrm{Q}=626.771$

CFI $=.976$

RMSEA $=.029$

Index; GDS = Geriatric Depression Scale; HCY = serum homocysteine; HgbA1c = serum hemoglobin A1c; RMSEA = Root Mean Square Error of Association. 


\section{References:}

419 relationship between behavioral and psychological symptoms (BPSD) and

420 caregiver well-being. Int Psychogeriatrics 2016; 28:1761-1774

421

422 2. Nobili A, Pasina L, Trevisan S, et al: Use and misuse of antipsychotic

423 drugs in patients with dementia in Alzheimer special care units. Int Clin

424 Psychopharmacol 2009; 24:97-104

426 3. Murman DL, Chen Q, Powell MC, et al: The incremental direct costs

427 associated with behavioral symptoms in AD. Neurology 2002; 59:1721-1729

429 4. de Oliveira AM, Radanovic M, de Mello PC, et al: Nonpharmacological

430 Interventions to Reduce Behavioral and Psychological Symptoms of Dementia: A

431 Systematic Review. Biomed Res Int 2015; 2015:218980

433 5. Toot S, Swinson T, Devine M, et al: Causes of nursing home placement for

434 older people with dementia: a systematic review and meta-analysis. Int

435 Psychogeriatrics 2017; 29:195-208

437 6. Lahey BB, Applegate B, Hakes JK, et al: Is there a general factor of 438 prevalent psychopathology during adulthood? J Abnormal Psychol 2012; $439 \quad 121: 971-977$ 
441 7. Caspi A, Houts RM, Belsky DW, et al: The $p$ factor: One general

442 psychopathology factor in the structure of psychiatric disorders? Clinical

443 Psychological Science 2014; 2:119-137

444

445 8. Caspi A, Moffitt TE. All for One and One for All: Mental Disorders in One

446 Dimension. Am J Psychiatry 2018; 175:831-844

448 9. Spearman C. General intelligence, objectively determined and measured.

449 Am J Psychology 1904; 15:201-293

451 10. Royall DR, Palmer RF, O'Bryant SE. Validation of a latent variable

452 representing the dementing process. J Alzheimer's Dis 2012; 30:639-649.

454 11. Royall DR, Palmer RF. "Executive functions" cannot be distinguished from 455 general intelligence: Two variations on a single theme within a symphony of latent 456 variance. Front Behav Neurosci 2014; 9:1-10

458 12. Royall DR, Palmer RF. $\delta$ scores identify subsets of "Mild Cognitive 459 Impairment" with variable conversion risks. J Alzheimer's Dis 2019; 70:199-210

461 13. O'Bryant SE, Waring SC, Cullum CM, et al. for the Texas Alzheimer's 462 Research Consortium. Staging dementia using Clinical Dementia Rating Scale 463 Sum of Boxes scores: A Texas Alzheimer's research consortium study. Arch 
464 Neurol, 2008; 65:1091-95

465

466 14. Royall DR, Palmer RF. A $\delta$ homolog for dementia case finding with

467 replication in Alzheimer's Disease Neuroimaging Initiative. J Alzheimer's Dis 2019;

$468 \quad 67: 67-79$

469

470 15. Palmer RF, Royall DR. Future dementia status is almost entirely explained

471 by the latent variable $\delta$ 's intercept and slope. J Alzheimer's Dis 2016; 49:521-529

472

473 16. Gavett BE, Vudy V, Jeffrey $M$, et al: The $\delta$ latent dementia phenotype in the

474 NACC UDS: Cross-Validation and Extension. Neuropsychology 2015; 29:344-352

475

476 17. John SE, Gurnani AS, Bussell C, et al: The Effectiveness and Unique

477 Contribution of Neuropsychological Tests and the $\delta$ Latent Phenotype in the

478 Differential Diagnosis of Dementia in the Uniform Data Set. Neuropsychology.

479 2016; 30: 946-960

480

481 18. Waring, S., O'Bryant, S.E., Reisch, J.S., et al, for the Texas Alzheimer's

482 Research Consortium. The Texas Alzheimer's Research Consortium longitudinal

483 research cohort: Study design and baseline characteristics. Texas Pub Health J 484 2008; 60:9-13. 
486 19. Arbuckle JL. Analysis of Moment Structures-AMOS (Version 7.0)

487 [Computer Program], SPSS, Chicago, 2006

489 20. Kaufer DI, Cummings JL, Ketchel P et al: Validation of the NPI-Q, a brief 490 clinical form of the Neuropsychiatric Inventory. J Neuropsychiatry Clin Neurosci $491 \quad 2000 ; 12: 233-239$.

493 21. Acevedo A, Krueger KR, Navarro E, et al: The Spanish translation and 494 adaptation of the Uniform Data Set of the National Institute on Aging Alzheimer's 495 Disease Centers. Alzheimer Dis Assoc Disord 2009; 23:102-109.

497 22. Sayegh P, Knight BG: Functional assessment and neuropsychiatric 498 inventory questionnaires: measurement invariance across Hispanics and non499 Hispanic whites. Gerontologist 2014; 54:375-386.

501 23. Folstein MF, Folstein SE, McHugh PR. Mini-mental state: a practical method for

502 grading the cognitive state of patients for the clinician. J Psychiatric Res 1975; $12: 189$ -

503198

504

505 24. Hughes, C.P., Berg, L., Danziger, W.L., Coben, L.A., Martin, R.L. (1982). A

506 new clinical scale for the staging of dementia. British Journal of Psychiatry. 140, $507 \quad$ 566-572. 
510 25. Yesavage JA, Brink TL, Rose TL, et al: Development and validation of a geriatric

511 depression screening scale: A preliminary report. J Am Geriatric Soc 1982; 29: 164-171

513 26. Hollingworth P, Hamshere ML, Moskvina V, et al: Four components describe

514 behavioral symptoms in 1,120 individuals with Late-Onset Alzheimer's Disease. J Am

515 Geriatric Soc 2006; 54:1348-1354

516

517 27. Palmer RF, Royall DR. Missing data? Plan on it! J Am Geriatric Soc. 2010;

518 58:S343-348

520 28. Schafer JL, Graham, JW Missing data: Our view of the state of the art. Psychol

521 Methods 2002; 7:147-177

522

523 29. Bollen KA, Long JS. Testing Structural Equation Models. Sage Publications,

524 Thousand Oaks, CA., 1993

525

526 30. Bentler PM. Comparative fit indexes in structural models. Psychol Bull 1990;

$527 \quad$ 107:238-246.

528

529 31. Wheaton B, Muthén B, Alwin DF, et al: Assessing reliability and stability in panel

530 models. In D.R.Heise (Ed.) Sociology Methodology San Francisco, CA: Jossey-Bass,

$531 \quad 1977$ 
533 32. Browne M, Cudeck R. Alternative ways of assessing model fit. In Testing

534 structural equation models, Bollen KA, Long JS, eds. Sage Publications, Thousand

535 Oaks, CA, pp. 136-162, 1993

536

537 33. Royall DR, Palmer RF, Matsuoka T, et al: Greater than the sum of its parts: $\delta$ can

538 be constructed from item level data. J Alzheimer's Dis 2016; 49:571-579

539

540 35. Bruen PD, McGeown WJ, Shanks MF, et al: Neuroanatomical correlates of

541 neuropsychiatric symptoms in Alzheimer's disease. Brain 2008; $131: 2455-2463$

543 35. Jellinger KA. Cerebral correlates of psychotic syndromes in neurodegenerative

544 diseases. J Cell Mol Med 2012; 16:995-1012

546 36. Jackson JH. Evolution and Dissolution of the Nervous System. In Selected

547 Writings of John Hughlings Jackson. Volume 2. Various Papers, Addresses and

$548 \quad$ Lectures. Arch Neuropsychiatry 1932; 28:967.

550 37. Jost BC, Grossberg GT. The evolution of psychiatric symptoms in

551 Alzheimer's disease: a natural history study. J American Geriatrics Soc. 1996;

$552 \quad 44: 1078-1081$.

553

554 38. Thompson $\mathrm{C}$, Brodaty $\mathrm{H}$, Trollor J, et al: Behavioral and psychological

555 symptoms associated with dementia subtype and severity. Int Psychogeriatrics.

$556 \quad 2010 ; 22: 300-3005$ 
558 39. Kazui $\mathrm{H}$, Yoshiyama K, Kanemoto $\mathrm{H}$, et al: Differences of Behavioral and

559 Psychological Symptoms of Dementia in Disease Severity in Four Major

560 Dementias. PLoS One 2016; 11:e0161092

561

562 40. Aalten $\mathrm{P}$, de Vugt ME, Jaspers $\mathrm{N}$, et al: The course of neuropsychiatric

563 symptoms in dementia. I. Findings from the two-year longitudinal Maasbed study.

564 Int J Geriatrics 2005; 20:523-530.

565

566 41. Hoffman BJ, Hansson SR, Mezey E, et al: Localization and dynamic regulation of

567 biogenic amine transporters in the mammalian central nervous system. Front

568 Neuroendocrinol 1998; 19:187-231

569

570 42. Lanari A, Amenta F, Silvestrelli G, et al: Neurotransmitter deficits in behavioural

571 and psychological symptoms of Alzheimer's disease. Mech Ageing Dev 2006; 127:158-

$572 \quad 165$

573

574 43. Staudt MD, Herring EZ, Gao K, et al: Evolution in the treatment of psychiatric

575 disorders: From psychosurgery to psychopharmacology to neuromodulation. Front

576 Neurosci 2019; 13:108 
bioRxiv preprint doi: https://doi.org/10.1101/813485; this version posted October 21,2019 . The copyright holder for this preprint (which was not certified by peer review) is the author/funder, who has granted bioRxiv a license to display the preprint in perpetuity. It is made available under aCC-BY-NC-ND 4.0 International license.

584

585 